\title{
TRATAMENTO CIRÚRGICO DA CISTICERCOSE DA FOSSA CRANIANA POSTERIOR
}

\author{
Pedro Garcia Lopes
}

A despeito de considerável progresso no diagnóstico da neurocisticercose, a terapêutica definitiva e completa erradicação dos parasitas ainda constituem problemas a resolver. A terapêutica medicamentosa, apesar do grande número de drogas experimentadas, não conduziu a resposta satisfatória. A terapêutica cirúrgica parece ser a que oferece o melhor resultado em determinados casos, ou seja, quando um cisto cisticercótico determina sintomatologia focal bem definida indicando lesão de zona cirùrgicamente accessível, ou quando ocasiona obstrução à circulação do líquido cefalorraqueano. Maior interêsse ocorre quando o processo localiza-se na fossa craniana posterior, em virtude da grave sintomatologia que ocasiona. Devido a alguns maus resultados e à grande mortalidade verificada não há, para êstes casos, uma conduta cirúrgica uniforme. A abordagem direta do processo foi considerada, e ainda o é por alguns autores, como o melhor método de tratamento cirúrgico. Ulteriormente, foram preconizadas intervenções cirúrgicas paliativas, com a única finalidade de derivar o trânsito do líquido cefalorraqueano para outras regiōes, intra ou extracranianas. Graças aos aperfeiçoamentos introduzidos, têm sido registrados, nos últimos anos, grande desenvolvimento nas técnicas de derivação, especialmente nas de tipo ventriculo-atrial e ventrículoperitonial.

É objetivo dêste trabalho demonstrar que, atualmente, os melhores métodos de tratamento cirúrgico para a cisticercose de fossa craniana posterior são as derivações extracranianas.

\section{MATERIAL E METODOS}

Foram estudados 70 pacientes com cisticercose de fossa craniana posterior, selecionados entre os 305 casos de neurocisticercose registrados no Serviço de Neurologia do Hospital das Clínicas da Faculdade de Medicina da Universidade de São Paulo,

Excerptos de tese de doutoramento defendida no Departamento de Neurologia e Comportamento (Clínica Neurológica) da Faculdade de Medicina do Norte do Paraná (Londrina, PR), em maio de 1970.

Nota do autor - Este trabalho foi realizado na Clínica Neurológica do Hospital das Clínicas da Faculdade de Medicina da Universidade de São Paulo, sob a orientação do Prof. Antonio Spina-França, constituindo a fase final de estágio de pósgraduação. Nas pessoas dos Professores Horácio M. Canelas e Rolando A. Tenuto, agradeço o treinamento recebido. 
no periodo de 1965 a $1968(70 / 305$, ou seja, 22,95\%). Os dados de identificação dos pacientes (idade, sexo, registro) encontram-se na tabela 1. Para a seleção dos casos foram exigidas três condiçōes: a) diagnóstico clínico-radiológico de afecção na fossa craniana posterior; b) diagnóstico etiológico de cisticercose comprovado pela positividade da reação de fixação do complemento no líquido cefalorraqueano e/ou mediante achado do parasita no ato cirúrgico ou durante necrópsia; c) pacientes submetidos a tratamento cirúrgico.

A metodologia adotada para o estudo dos casos compreendeu a apreciação das caracteristicas clínicas, dos exames complementares, do tratamento e da evolução.

Visto que o material utilizado neste trabalho pertence a um grupo particular de neurocisticercose, o mesmo não serve para caracterizar as formas clínicas, mas sim para estudar apenas os quadro clínicos resultantes da cisticercose de fossa posterior (Tabela 2). Assim, a hipertensão intracraniana foi encontrada em 98,57\%

\begin{tabular}{|c|c|c|c|c|c|c|c|c|c|}
\hline Caso & Nome & Idade & Sexo & Registro & Caso & Nome & Idade & Sexo & Registro \\
\hline 1 & VMF & 35 & $\mathbf{F}$ & 814.133 & 36 & TRM & 23 & $\mathbf{F}$ & 400.372 \\
\hline 2 & JP & 9 & M & 30.580 & 37 & ATO & 35 & $\mathbf{M}$ & 287.231 \\
\hline 3 & $\mathrm{AAB}$ & 40 & M & 405.227 & 38 & EF & 26 & $\mathbf{M}$ & 454.831 \\
\hline 4 & EVC & 19 & $F$ & 503.127 & 39 & AMM & 28 & $\mathbf{F}$ & 399.633 \\
\hline 5 & PRN & 43 & $\mathbf{M}$ & 146.440 & 40 & MDA & 47 & $\mathbf{M}$ & 452.201 \\
\hline 6 & NAM & 20 & $F$ & 409.810 & 41 & $\mathrm{OD}$ & 36 & $\mathbf{M}$ & 496.807 \\
\hline 7 & CPM & 30 & F & 484.998 & 42 & $\mathrm{DZ}$ & 18 & $\mathbf{F}$ & 640.745 \\
\hline 8 & VP & 30 & $\mathbf{M}$ & 584.095 & 43 & IAP & 28 & $\mathbf{F}$ & 664.447 \\
\hline 9 & FM & 36 & $\mathbf{M}$ & 735.552 & 44 & VA & 26 & $\mathbf{M}$ & 555.807 \\
\hline 10 & AGS & 31 & $\mathbf{M}$ & 783.230 & 45 & DRP & 13 & $\mathbf{F}$ & 831.632 \\
\hline 11 & $\mathrm{AAC}$ & 6 & M & 755.628 & 46 & $\mathrm{AC}$ & 28 & $\mathbf{M}$ & 511.126 \\
\hline 12 & MAOM & 11 & $\mathbf{F}$ & 784.183 & 47 & $\mathrm{AV}$ & 38 & $\mathbf{M}$ & 584.454 \\
\hline 13 & IMR & 26 & $\mathbf{F}$ & 692.113 & 48 & CML & 40 & $\mathbf{F}$ & 645.516 \\
\hline 14 & JAL & 25 & M & 513.131 & 49 & VAR & 35 & $\mathbf{F}$ & 679.778 \\
\hline 15 & JRS & 33 & $\mathbf{M}$ & 488.210 & 50 & IMF & 31 & $\mathbf{F}$ & 567.430 \\
\hline 16 & LAS & 17 & $F$ & 403.967 & 51 & MHM & 44 & $F$ & 774.520 \\
\hline 17 & $\mathrm{ZMG}$ & 20 & $F$ & 713.392 & 52 & LMM & 26 & M & 884.827 \\
\hline 18 & $\mathbf{J M}$ & 37 & M & 208.065 & 53 & AMP & 46 & $\mathbf{F}$ & 751.409 \\
\hline 19 & JM & 40 & M & 179.047 & 54 & $\mathrm{NMH}$ & 35 & $F$ & 614.272 \\
\hline 20 & ATM & 39 & $\mathbf{M}$ & 79.647 & 55 & LFB & 8 & $\mathbf{F}$ & 746.551 \\
\hline 21 & JJO & 27 & $\mathbf{M}$ & 738.581 & 56 & MBS & 38 & $\mathbf{M}$ & 779.267 \\
\hline 22 & ML & 34 & $\mathbf{M}$ & 688.295 & 57 & AAR & 36 & $\mathbf{M}$ & 775.187 \\
\hline 23 & JB & 42 & $\mathbf{M}$ & 48.676 & 58 & PIO & 29 & $\mathbf{M}$ & 654.458 \\
\hline 24 & MGAR & 27 & $F$ & 370.898 & 59 & TL & 47 & $F$ & 614.510 \\
\hline 25 & AEM & 30 & $\mathbf{F}$ & 367.899 & 60 & MGS & 19 & $\mathbf{F}$ & 642.364 \\
\hline 26 & AA & 34 & $\mathbf{M}$ & 388.110 & 61 & MML & 40 & $\mathbf{M}$ & 583.977 \\
\hline 27 & PG & 27 & $\mathbf{M}$ & 154.475 & 62 & DLR & 21 & $\mathbf{M}$ & 711.939 \\
\hline 28 & FO & 25 & M & 196.297 & 63 & JOF & 39 & $\mathbf{F}$ & 887.069 \\
\hline 29 & CBJ & 20 & $\mathbf{M}$ & 440.524 & 64 & JED & 13 & $\mathbf{M}$ & 851.345 \\
\hline 30 & OGS & 32 & $F$ & 716.503 & 65 & MAF & 13 & $\mathbf{F}$ & 814,064 \\
\hline 31 & MA & 8 & $\mathbf{F}$ & 472.496 & 66 & $\mathrm{JCD}$ & 4 & $\mathbf{F}$ & 871.809 \\
\hline 32 & JL & 36 & $\mathbf{M}$ & 533.934 & 67 & DS & 32 & $\mathbf{M}$ & 813.669 \\
\hline 33 & IAM & 30 & $F$ & 406.537 & 68 & MLV & 18 & $\mathbf{F}$ & 832.289 \\
\hline 34 & MRM & 52 & $\mathbf{F}$ & 452.904 & 69 & $\mathbf{L V}$ & 30 & $\mathbf{F}$ & 848.996 \\
\hline 35 & HMBP & 23 & $\mathbf{F}$ & 436.182 & 70 & $\mathrm{JC}$ & 11 & $\mathbf{M}$ & 874.385 \\
\hline
\end{tabular}

Tabela 1 - Dados de identificação dos pacientes estudados: idade em anos. 
Sintomatologia

N. ${ }^{\circ}$ casos

Hipertensão intracraniana

Distúrbios psiquicos

Convulsōes (generalizadas, 11; focais, 7)

Sinais de lesão piramidal (unilaterais, 7; bilaterais, 3)

Sintomas cerebelares - apendiculares (unilaterais, 1; bilaterais, 1)

- axiais

- globais

Comprometimento de nervos cranianos:

V (unilateral)

VI (unilateral, 6; bilateral, 2)

VII (unilateral)

VIII (unilateral, 6; bilateral, 2)

Tabela 2 - Sinais e sintomas clínicos encontrados nos 70 casos estudados.

dos casos, e isto explica a alta incidência de distúrbios visuais $(71,43 \%$ dos casos), consequiente a papiledema. Convulsōes não foram muito freqüentes (18 casos), predominando as crises generalizadas (11 casos). Entre os nervos cranianos, os mais lesados foram os motores oculares, o acústico e o trigêmio. Quadro cerebelar ocorreu em 26 casos, havendo predomínio da síndrome axial (15 casos). Manifestações psíquicas foram encontradas em apenas 10 casos, o mesmo ocorrendo com a sindrome piramidal. Distúrbios sensitivos estavam presentes em somente 8 casos. Considerando-se o faio de que o cisticerco pode provocar reações locais e/ou gerais, torna-se dificil saber quais são as síndromes que decorrem da hipertensão intracraniana e quais as que são causadas pela parasitose em sí.

O diagnóstico dos casos utilizados neste trabalho foi feito, em 35 dêles, pela positividade da reação de fixação de complemento no liquido cefalorraqueano; em 20 pelo achado cirúrgico; em 9 pela associação de ambos. Apenas em 5 casos não foi o diagnóstico estabelecido em vida. A positividade da reação de fixaçāo do complemento no líquido cefalorraqueano foi de $71,43 \%$, permitindo o diagnóstico de segurança. Das outras alterações encontradas no liquido cefalorraqueano destacam-se: pleocitose discreta em $68,25 \%$ dos casos, com a presença de eosinófilos em $\mathbf{3 6 , 5 1 \%}$; hiperproteinorraquia em $58,73 \%$ dos casos. A reação de fixação do complemento no sôro também foi estudada e mostrou-se positiva em $71,43 \%$ dos casos. Eletrencefalograma com alterações foi encontrado em 77,5\% dos casos: alterações difusas em 15 casos e focais em 16; no entanto, dos que apresentavam disritmia focal, apenas 5 tinham convulsões. As radiografias simples de crânio mostraram sinais de hipertensão intracraniana em $60 \%$ dos casos e calcificações em $5,71 \%$. A angiografía pela carótida, em $86,36 \%$ dos casos, localizou o processo na fossa craniana posterior, evidenciando sinais indiretos de dilatação ventricular. A iodoventriculografia mostrou-se alterada em $96,43 \%$ dos casos, revelando alteracōes anatômicas que permitiam situar o processo responsável pela hipertensão intracraniana ao nivel da fossa craniana posterior. 
Todos os casos foram submetidos a tratamento cirúrgico, segundo técnicas diversas (Tabela 3). Depenđendo do caso, várias foram as técnicas empregadas, assim agrupáveis: 1) craniectomia de fossa posterior (Tabela 4), quando o trânsito do liquido cefalorraqueano foi normalizado, seja pela retirada de cisticerco, seja desfazendo bloqueio inflamatório; 2) derivações intracranianas (Tabela 5), quando o trânsito do liquido cefalorraqueano foi derivado para outras regiões do próprio continente crânio-vertebral (abertura da lâmina terminal, derivação transcalosa, derivação ventrículo-cisternal); 3) derivações extracranianas (Tabela 6), ventrículo-atrial ou ventriculo-peritonial.

$\begin{array}{lll}\text { Tipo de cirurgia } & \text { N.o casos } & \text { Percentagem }\end{array}$

Craniectomia de fossa posterior $\quad 33$ ( 1 a 33) $47,14 \%$

Derivaçāo intracraniana $\quad 17$ (34 a 50) 24,29\%

Derivação extracraniana $\quad 20(51$ a 70$) \quad 28,57 \%$

Tabela 3 - Tratamentos cirúrgicos realizados nos 70 casos estudados.

Craniectomias

N.D casos

Percentagem

$\begin{array}{lrr}\text { Retirada de cisticercos } & 25 & 35,71 \% \\ \text { Permeabilização de bloqueios } & 8 & 11,43 \%\end{array}$

Tabela 4 - Craniectomias de fossa posterior realizadas em 33 pacientes.

Derivações intracranianas $\quad$ N.o casos $\quad$ Percentagem

$\begin{array}{llrr}\text { Abertura da lâmina terminal } & 8 & 11,43 \% \\ \text { Transcalosa } & 3 & 4,29 \% \\ \text { Ventriculo-cisternal } & 6 & 8,57 \%\end{array}$

Tabela 5 - Derivaçöes intracranianas realizadas em 17 pacientes. 
R E S U L T A D O S

Sendo dificil fazer o seguimento pós-operatório de pacientes que apresentam, na fase pré-operatória, quadros clínicos polimorfos como são os determinados pela neurocisticercose, o estudo da evolução orientou-se para a principal das sindromes observadas, a de hipertensão intracraniana que $69 \%$ dos pacientes apresentavam e que, em 21 dêles $(30 \%)$, era a única manifestação clínica da moléstia. Considerando as condições dos pacientes (melhorada, inalterada e piorada), os resultados cirúrgicos foram tabulados sendo subdivididos de acôrdo com o tipo de cirurgia empregada e conforme o tipo de patologia encontrada. As tabelas 7,8 e 9 mostram os resultados verificados 10,20 e 30 dias após as intervençōes cirúrgicas. As tabelas 10 e 11 condensam os resultados globais no seguimento pós-operatório durante $\mathbf{3 0}$ dias e as complicaçōes, neurológicas ou não, observadas nêste periodo.

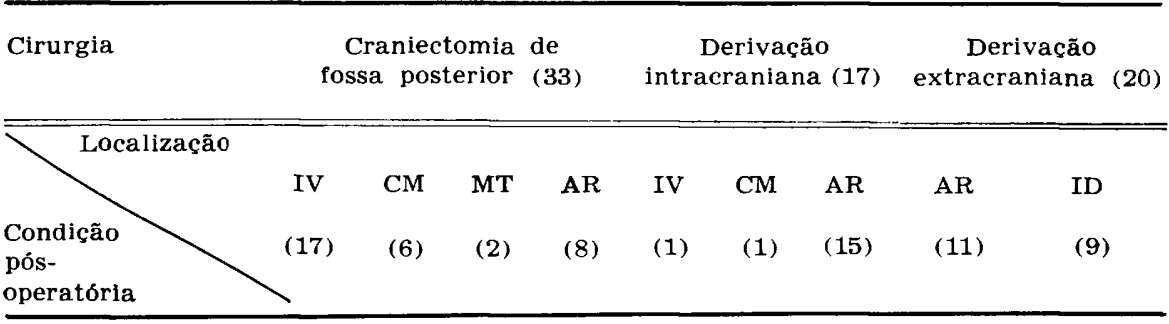

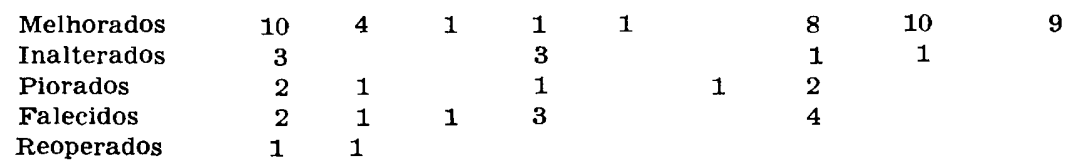

Tabela 7 - Evolução dos casos até o $100^{\circ}$ dia de pós-operatório (70 casos). Legenda: $I V=$ cisticerco de IV ventrículo; $C M=$ cisticerco de cisterna magna; $M T=$ cisticercos múltiplos; $A R=$ aracnoidite; $I D=$ indeterminado. Os números colocados nos parênteses indicam o número de pacientes acompanhados nêsse perído.

\begin{tabular}{lccc}
\hline Cirurgia & $\begin{array}{c}\text { Craniectomia de } \\
\text { fossa posterior (25) }\end{array}$ & Derivação & Derivação \\
& intracraniana (13) extracraniana
\end{tabular}

$\begin{array}{lllllllllll}\text { Localização } & \text { IV } & \mathrm{CM} & \mathrm{MT} & \mathrm{AR} & \mathrm{IV} & \mathrm{CM} & \mathrm{AR} & \mathrm{IV} & \text { AR } & \text { ID } \\ \begin{array}{l}\text { Condição } \\ \text { pós- } \\ \text { operatória }\end{array} & \text { (14) } & \text { (5) } & \text { (1) } & \text { (5) } & \text { (1) } & \text { (1) } & \text { (11) } & \text { (1) } & \text { (11) } & \text { (9) }\end{array}$

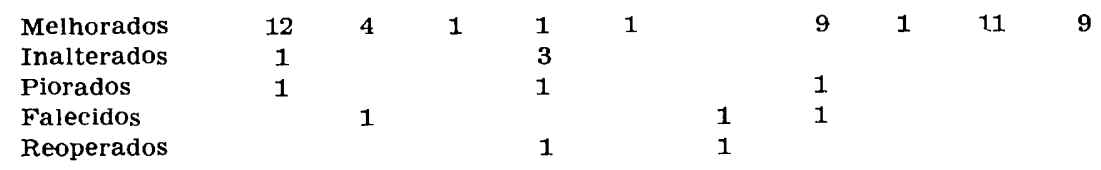

Tabela 8 - Evolução dos casos do $11 .^{\circ}$ ao $20 .^{\circ}$ dia de pós-operatório (59 casos). Legenda: vide tabela 7 . 
Os dados gerais de evolução dos pacientes até 5 anos de seguimento pós-operatório (P.O.) são encontrados nas tabelas discriminativas de cada periodo (Tabelas 12 a 17). Algumas particularidades da evoluçāo são relatadas a seguir. No periodo de 1 a 10 dias, dois casos foram reoperados por apresentarem hipertensão intracraniana; um (caso 30 ) foi submetido à derivação ventrículo-atrial no $2 .^{\circ}$ día do P.O. e outro (caso 20) à revisão de cirurgia no 9.0 dia do P.D. No periodo de 11 a 20 dias, foram efetuadas duas reoperaçôes: uma (caso 32 ) com abertura da lâmina terminal, por ocorrer hipertensão intracraniana no $20 .^{\circ}$ dia do P.O.; outra (caso 35) para revisão de derivação ventrículo-cisternal com retirada da sonda, por terem surgido sinais de meningite no 16.0 dia do P.O. No periodo de 21 a 30 dias no $28 .^{\circ}$ dia do P.O., surgiram, no caso 51, sinais de hipertensão intracraniana, sendo verificado que o sistema de derivação funcionava normalmente, tendo o paciente melhorado, ulteriormente, com tratamento medicamentoso. No periodo de 1 a 3 mêses, o caso 31 continuava a piorar e foi submetido à derivação transcalosa no 31.0 dia do P.O.; o caso 33 recomeçou a apresentar hipertensão intracraniana, sendo revista a cirurgia no $45.0^{\circ}$ dia de P.O., normalizando-se o trânsito do liquido cefalorraqueano e, não havendo melhora mesmo assim, foi feita derivação transcalosa 20 dias após; o caso 53 apresentou meningite purulenta sendo retirado o sistema de derivaçāo; o caso 58 piorou e um exame radiológico de crânio mostrou bloqueio parcial do cateter ventricular por lipiodol, porém o quadro clínico regrediu com tratamento medicamentoso; o caso 59 reapresentou hipertensão intracraniana tendo sido revista a cirurgia, que mostrou obstrução do cateter atrial; o caso 61 teve hipotensão intracraniana, sendo retirado o sistema de derivação. No periodo de 6 a 12 mêses, o caso 10 apresentou, no $7 .^{\circ}$ mês, sinais de hipertensão intracraniana, melhorando com tratamento medicamentoso.

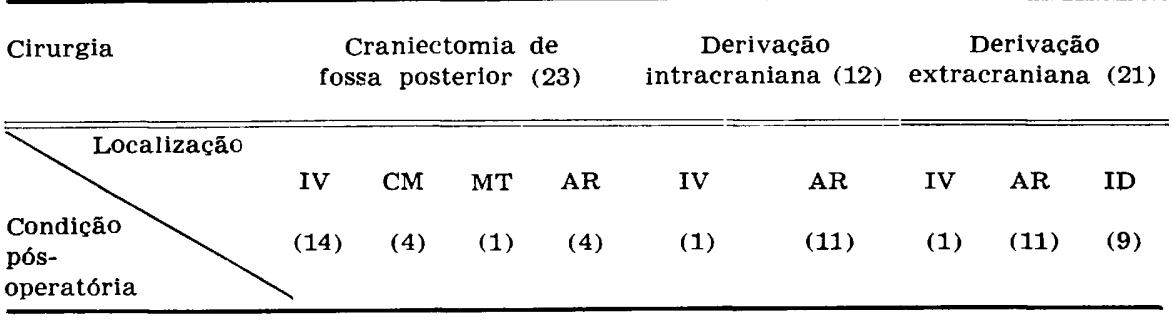

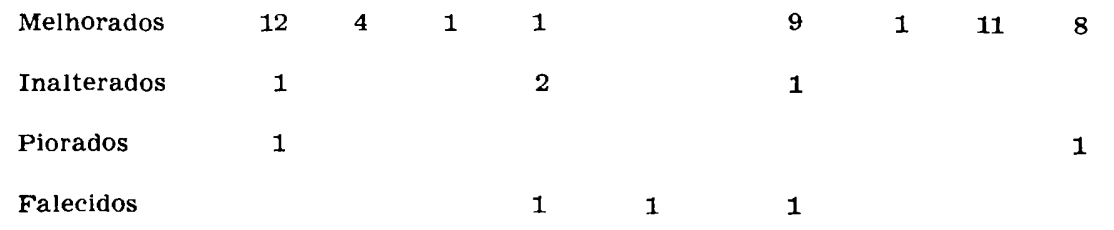

Tabela 9 - Evotuç̄o dos casos do $21 .^{\circ}$ ao $30 .^{\circ}$ de pós-operatório (56 casos). Legenda: vide tabela 7 . 


\begin{tabular}{lccc} 
Cirurgia & $\begin{array}{c}\text { Craniectomia de } \\
\text { fossa posterior }\end{array}$ & $\begin{array}{c}\text { Derivação } \\
\text { intracraniana } \\
\text { Condição } \\
\text { pós- } \\
\text { operatória }\end{array}$ & $\begin{array}{c}\text { Derivação } \\
\text { extracraniana } \\
(21)\end{array}$ \\
\hline$\quad$ Melhorados & 18 & 9 & 20 \\
$\quad \begin{array}{l}\text { Inalterados } \\
\text { Piorados }\end{array}$ & 3 & 1 & 1 \\
$\quad$ Falecidos & 1 & 8 & \\
Reoperados & 9 & 1 & \\
\hline
\end{tabular}

Tabela 10 - Evoluçáo geral dos casos ate o $300^{\circ}$ dia de pós-operatório (70 casos).

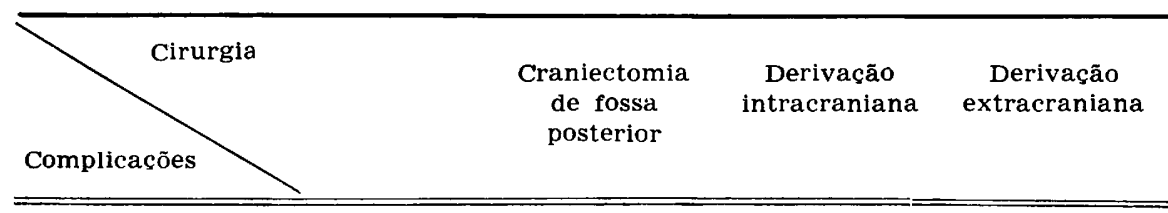

Abaulamento da região operada

Fistula pela incisāo

Convulsões

2

Meningite

Broncopneumonia

Diabete insipido

Atelectasia pulmonar

Hemiparesia transitória

1

1

2

1

1

1

2

Tabela 11 - Complicações pós-operatórias.

$\begin{array}{rlcc}\text { Cirurgia } & \text { Craniectomia de } & \text { Derivação } & \text { Derivação } \\ \text { fossa posterior } & (22) & \text { intracraniana (10) } & \text { extracraniana }\end{array}$

fossa posterior (22) intracraniana (10) extracraniana (21)

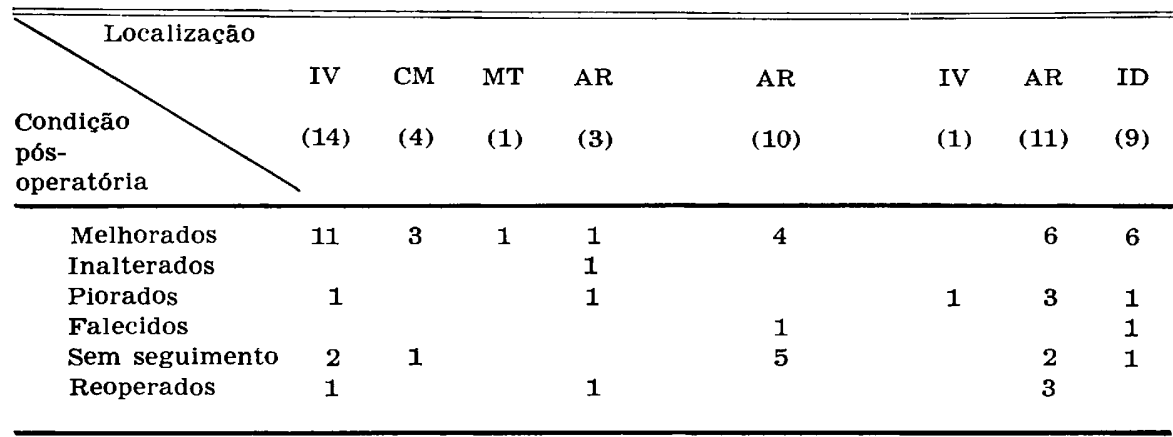

Tabela 12 - Evoluçāo dos casos de 1 a 3 mêses de pós-operatório (53 casos). Legenda: vide tabela 7 . 


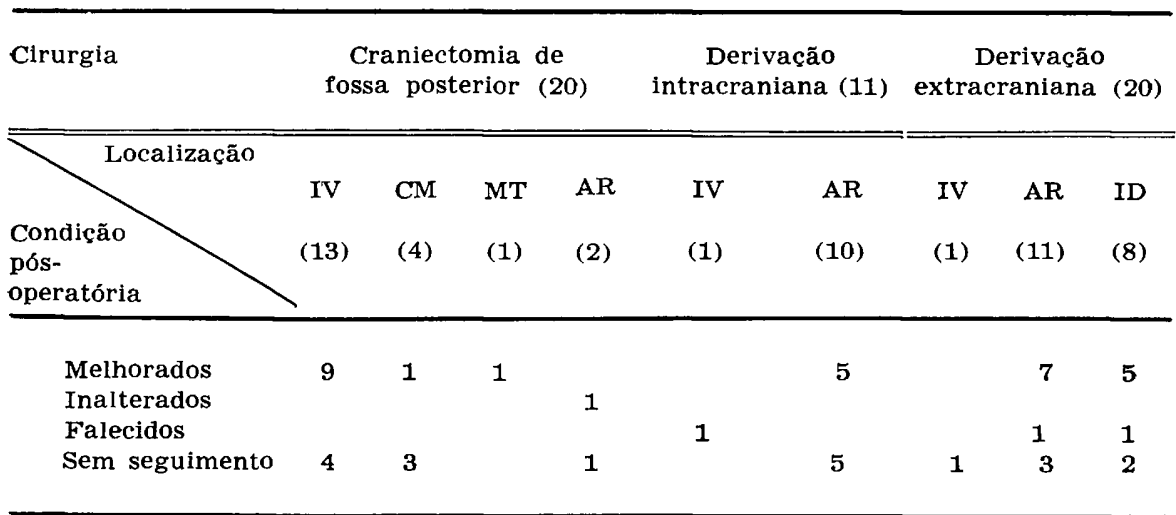

Tabela 13 - Evoluৎ̣̄o dos casos de 3 a 6 mêses de pós-operatório (51 casos). Legenda: vide tabela 7 .

\begin{tabular}{lccc}
\hline Cirurgia & $\begin{array}{c}\text { Craniectomia de } \\
\text { fossa posterior }(20)\end{array}$ & $\begin{array}{c}\text { Derivação } \\
\text { intracraniana (10) extracraniana (18) }\end{array}$
\end{tabular}

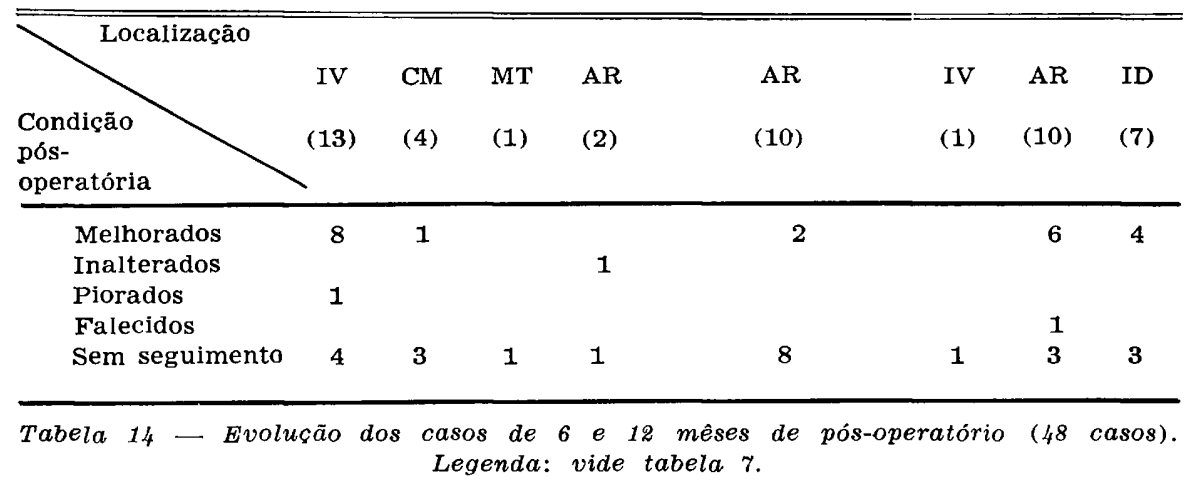

$\begin{array}{lccc}\text { Craniectomia de } & \text { Derivação } & \text { Derivação } \\ \text { fossa posterior }(20) & \text { intracraniana (10) } & \text { extracraniana (17) }\end{array}$

\begin{tabular}{|c|c|c|c|c|c|c|c|c|}
\hline $\begin{array}{l}\text { Condição } \\
\text { pós- } \\
\text { operatória }\end{array}$ & $\begin{array}{l}\text { IV } \\
\text { (13) }\end{array}$ & $\begin{array}{l}\text { CM } \\
\text { (4) }\end{array}$ & $\begin{array}{l}\text { MT } \\
\text { (1) }\end{array}$ & $\begin{array}{l}\text { AR } \\
(2)\end{array}$ & $\begin{array}{l}\text { AR } \\
(10)\end{array}$ & $\begin{array}{l}\text { IV } \\
\text { (1) }\end{array}$ & $\begin{array}{l}\text { AR } \\
(9)\end{array}$ & $\begin{array}{l}\text { ID } \\
\text { (7) }\end{array}$ \\
\hline Melhorados & 9 & 1 & & & 1 & & 5 & \\
\hline Sem seguimento & 4 & 3 & 1 & 2 & 9 & 1 & 4 & 7 \\
\hline Tabela $15-E v o$ & $\tilde{a}$ & & $\begin{array}{l}s \quad d \\
e n d a\end{array}$ & $\begin{array}{c}1 \quad a \\
\text { vide }\end{array}$ & $\begin{array}{l}\text { s de } \\
7 .\end{array}$ & atóri & $(47$ & (asos). \\
\hline
\end{tabular}




$\begin{array}{rlcr}\text { Craniectomia de } & \text { Derivacão } & \text { Derivação } \\ \text { Cirurgia } & \text { fossa posterior }(20) & \text { intracraniana (10) } & \text { extracraniana }\end{array}$

\begin{tabular}{lllllllll}
\hline Localização & IV & CM & MT & AR & AR & IV & AR & ID \\
$\begin{array}{l}\text { Condição } \\
\text { pós- } \\
\text { operatória }\end{array}$ & (13) & (4) & (1) & (2) & (10) & (1) & (9) & (7) \\
\hline
\end{tabular}

$\begin{array}{lllll}\text { Melhorados } & 6 & 1 & 1 & 2\end{array}$

Falecidos

1

$\begin{array}{lllllllll}\text { Sem seguimento } & 7 & 3 & 1 & 2 & 9 & 1 & 6 & 7\end{array}$

Tabela 16 - Evolução dos casos de 2 a 3 anos de pós-operatório (47 casos). Legenda: vide tabela 7 .

Cirurgia

Craniectomia de

Derivação

Derivação

fossa posterior (20) intracraniana (10) extracraniana

(16)

\begin{tabular}{llllllllll}
\hline Localizaçãc & IV & $\mathrm{CM}$ & $\mathrm{MT}$ & $\mathrm{AR}$ & $\mathrm{AR}$ & $\mathrm{IV}$ & $\mathrm{AR}$ & $\mathrm{ID}$ \\
$\begin{array}{l}\text { Condição } \\
\text { pós- } \\
\text { operatória }\end{array}$ & (13) & (4) & (1) & (2) & (10) & (1) & (8) & (7) \\
\hline
\end{tabular}

Melhorados

3

1

$\begin{array}{lllllllll}\text { Sem seguimento } & 10 & 4 & 1 & 2 & 10 & 1 & 7 & 7\end{array}$

ŕabela 17 - E'volução dos casos de 3 a 5 anos de pós-operatório (46 casos). Legenda: vide tabela $\%$.

A tabela 18, mostra um apanhado geral de tôda a evolução até 5 anos. Dos 21 pacientes que faleceram durante a internação, 17 foram submetidos à necrópsia (casos 15, 16, 18, 19, 20, 23, 27, 28, 31, 35, 36, 37, 40, 46, 47, 48, 61). Todos apresentavam neurocisticercose difusa; em 9 foi encontrada aracnoidite, 4 apresentavam vários eisticercos e, em 4, os dois problemas se associavam. As causas que provocaram a morte estāo relacionadas na tabela 19 . 


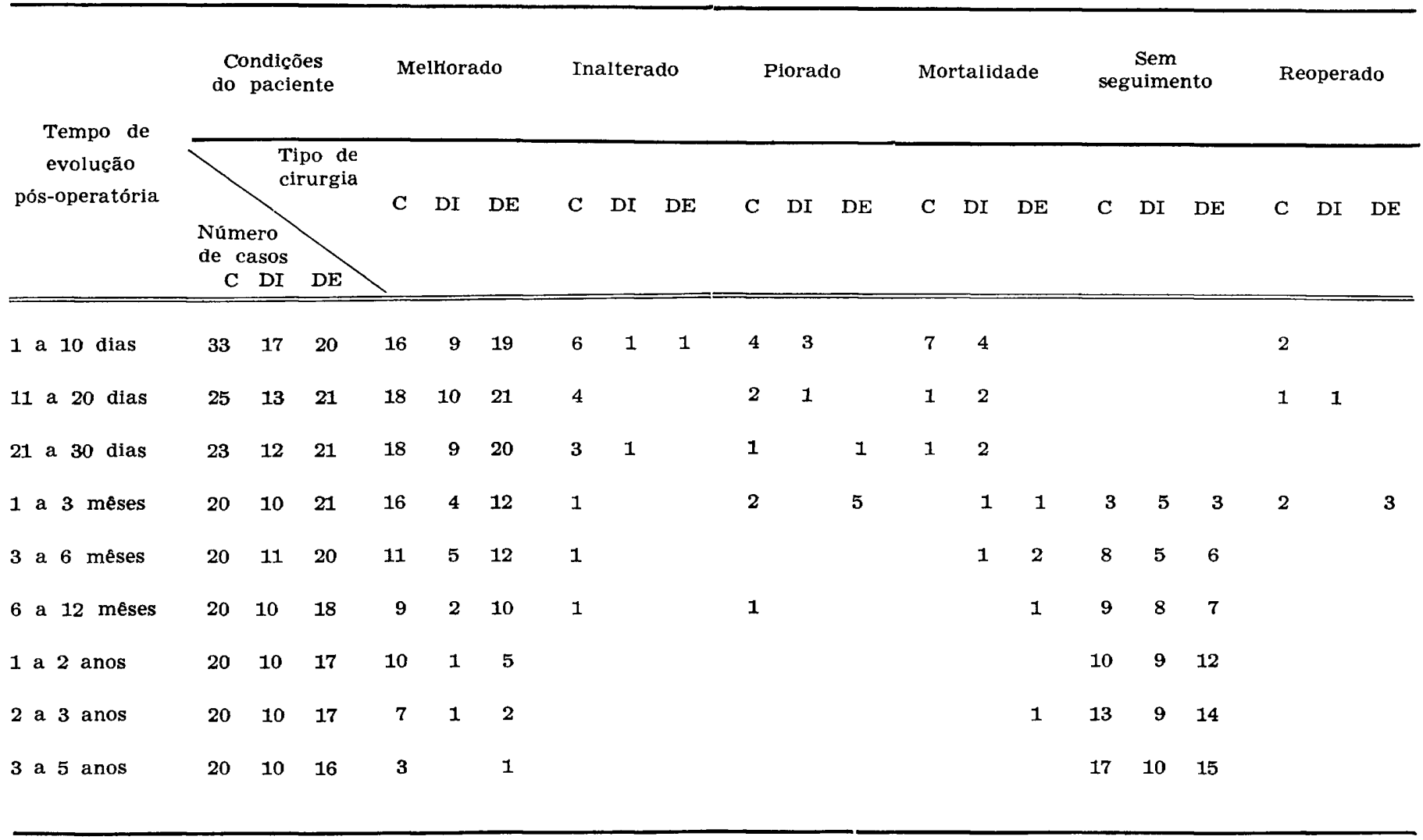

Tabela 18 - Avaliação total da evolucão dos casos até 5 anos de pós-operatório. Legenda: C = craniectomia de fossa posterior; $D \dot{I}=$ derivacão intracraniana; $D E=$ derivação extracraniana. 


\begin{tabular}{|c|c|c|c|}
\hline Causa & $\begin{array}{l}\text { Craniectomia } \\
\text { de fossa } \\
\text { posterior }\end{array}$ & $\begin{array}{l}\text { Derivação } \\
\text { intracraniana }\end{array}$ & $\begin{array}{l}\text { Derivação } \\
\text { extracraniana }\end{array}$ \\
\hline Hipertensão intracraniana & 6 & 2 & \\
\hline Meningite & 1 & 3 & \\
\hline $\begin{array}{l}\text { Hipertensão intracraniana e } \\
\text { meningite }\end{array}$ & 1 & 1 & \\
\hline Broncopneumonia & & 2 & 1 \\
\hline
\end{tabular}

Tabela 19 - Fatôres que provocaram a morte nos casos com necrópsia.

\section{O M E T T R I O S}

Os casos analisados neste trabalho estão, em parte, englobados no material de Canelas ${ }^{11}$. Os dados relativos ao sexo (discreto predomínio no sexo masculino) e idade $(64,29 \%$ dos pacientes tinham entre 21 e 40 anos), tempo de evolução da doença (menos de um ano em 48,57\% dos casos) e procedência $(58,57 \%$ dos casos provinham da zona rural), ora encontrados coincidem com os resultados dêsse autor, evidenciando que a amostra utilizada no presente estudo não difere do material global da qual foi retirada, dela sendo, portanto, representativa.

O cisticerco pode provocar reações locais (granulomas) e reações gerais, sendo que estas são mais freqüentes nas meninges, podendo as leptomeningites decorrerem de reações locais e/ou difusas. Na fossa craniana posterior costuma ocorrer um processo de leptomeningite altamente proliferativa, formando-se denso tecido de granulação, que logo tende à fibrose e pode determinar distúrbios na circulação do líquido cefalorraqueano ${ }^{29}$.

O polimorfismo sintomatológico da neurocisticercose tem originado diversidade nas classificações das formas clínicas ${ }^{12,22,24,30,35}$. Nos casos de cisticercose da fossa craniana posterior, a síndrome de hipertensão intracraniana é a mais comum ${ }^{23},{ }^{36}$, os últimos nervos cranianos podendo ser comprometidos associadamente, especialmente os responsáveis pela motricidade extrínseca do ôlho, bem como os nervos auditivo e trigêmio $5,9,19,26$; é freqüente o acometimento cerebelar (axial e/ou apendicular) ${ }^{6}$.

A positividade da reação de fixação do complemento no líquido cefalorraqueano é o elemento de segurança para o diagnóstico de afecção ${ }^{34}$. Em geral, ela é positiva em $2 / 3$ dos casos ${ }^{34}$. Pôsto que não há positividade da reação de fixação do complemento no líquido cefalorraqueano em todos os casos, outros exames paraclínicos são utilizados, pois podem fornecer dados que auxiliam o diagnóstico. Assim, no líquido cefalorraqueano, pode ocorrer 
pleocitose discreta com presença de eosinófilos e aumento da concentração de proteínas totais ${ }^{34,41}$. O perfil eletroforético das proteínas costuma estar alterado, havendo aumento da globulina gama ${ }^{33}$. A reação de fixação do complemento no sôro também deve ser realizada, pois apesar de sua positividade não apontar especificamente cisticercose do sistema nervoso, pode ser indicativa de uma infestação sistêmica. Proctor e col. ${ }^{28}$ tiveram positividade de $85 \%$ em casos de neurocisticercose. Raramente o eletrencefalograma é normal, quase sempre apresentando um traçado comum na síndrome de hipertensão intracraniana, ou seja, sofrimento cerebral difuso (ondas lentas) ${ }^{25}$. As disritmias focais indicam com grande ênfase a presença de convulsões ${ }^{25}$. Os exames radiológicos fornecem poucos dados para o diagnóstico etiológico ${ }^{42}$. As radiografias simples de crânio podem mostrar sinais de hipertensão intracranianua e/ou caicificações. Estas não são comuns, aparecendo em $4 \%$ dos casos aproximadamente, contrastando com os sinais de hipertensão intracraniana, presentes em tôrno de $70 \%$ dos casos ${ }^{15}, 19$. Os exames radiológicos contrastados têm grande valor no diagnóstico topográfico. Dêstes, são importantes a angiografia pela carótida e a iodoventriculografia: a angiografia pela carótida, nos processos de fossa craniana posterior que provocam hidrocefalia interna, mostra sinais indiretos de dilatação ventricular; a iodoventriculografia, permite localizar o processo na fossa craniana posterior ${ }^{3}$, embora raramente evidencie o parasita ${ }^{14}$.

O tratamento cirúrgico parece ser o único realmente efetivo para a neurocisticercose, especialmente como paliativo ${ }^{21}$. Segundo Goñi ${ }^{19}$, Cabiesis indica a cirurgia na cisticercose do sistema nervoso central quando é comprovada a obstrução do trânsito do líquido cefalorraqueano e em casos de epilepsia focal incontrolável por medicamentos. Com a extirpação do parasita, em casos de neurocisticercose supratentorial, tem sido conseguida, em certas ocasiões, regressão total da sintomatologia ${ }^{7}, 10,31$. Nos casos de fossa craniana posterior, a mortalidade cirúrgica é muito grande, qualquer que seja a técnica empregada. Iizuka ${ }^{21}$, em revisão da literatura, encontrou 30 a $40 \%$ de mortalidade total, maior que a relatada por Obrador ${ }^{27}$ em casos de tumores benígnos de fossa posterior. Stepién ${ }^{36}$, em casos de leptomeningite crônica e ependimite, teve mortalidade de $67,6 \%$. Em virtude disto, há grande discussão em tôrno de qual seja o melhor método cirúrgico.

A abordagem direta, realizada mediante craniectomia de fossa posterior, era largamente usada. A mortalidade oscila conforme os vários autores, de $18,1 \%$ a $50 \%$ nos casos de cisto único e de $44 \%$ a $71,43 \%$ nos casos de cisticerco racemoso e cisticercose difusa ${ }^{6,8,38,39}$. Os que atualmente defendem êste tipo de cirurgia, indicam-no principalmente nos casos de cisticerco de IV ventrículo, alegando a grande possibilidade do mesmo ser único e, da extirpação, sobrevir a cura ${ }^{4,37}$. Porém outros, argumentando ser a neurocisticercose um processo difuso, geralmente com aracnoidite crônica associada ${ }^{1,16,40}$, propõem outros métodos cirúrgicos. Hainning e Hainning ${ }^{20}$ relatam um caso de extirpação de cisticerco de IV ventriculo, em que a hipertensão intracraniana reapareceu 18 mêses após a cirurgia e a necrópsia mostrou aracnoidite crônica. Além disto, a retirada do cisto geralmente provoca 
meningite pós-operatória, provàvelmente pela rotura do mesmo. Canelas e col. ${ }^{13}$ publicaram 7 casos de cisticerco localizado no recesso lateral da cisterna pontina, dando uma síndrome de ângulo ponto-cerebela . Nêstes casos, a abordagem direta torna-se obrigatória.

As derivações intracranianas estão, progressivamente, tendo uso limitado nos casos de neurocisticercose. Isto porque, sendo esta um processo difuso, provoca bloqueio em diversos níveis do trajeto do liquido cefalorraqueano e impede a reabsorção do mesmo. Valladares e Poblete ${ }^{40}$ relataram dois casos submetidos a tal tratamento, tendo os pacientes falecido alguns dias após a cirurgia. Simms e col. ${ }^{32}$, revendo os casos de neurocisticercose publicados nos EE.UU., encontraram apenas um que fôra submetido à derivação intracraniana, tendo o paciente falecido no pós-operatório; outro, que havia sido submetido à craniectomia de fossa posterior associada à derivação intracraniana, teve que ser reoperado por apresentar hipertensão intracraniana. Forjaz e col. ${ }^{17}$ vêm usando uma nova técnica para ventriculostomia através do hipotálamo; entretanto, para os casos de neurocisticercose podem vir a ocorrer os mesmos problemas das outras derivações intracranianas.

As derivações extracranianas, que afastam os problemas atinentes à dificuldade de absorção do líquido cefalorraqueano provocada pela neurocisticercose, constituem-se no melhor método cirúrgico ${ }^{1}$. A principal contraindicação das derivações extracranianas é o processo infeccioso que pode ocorrer no pós-operatório. Almeida e col. ${ }^{2}$ em 184 derivações ventrículo-peritoniais, tiveram 39 complicações infecciosas. Forrest e col. ${ }^{18}$ em revisão de 455 casos de derivação ventrículo-atrial, encontraram 44 casos em que os pacientes haviam falecido em conseqüência de complicações do sistema de derivação (hipertensão intracraniana e septicemia). A causa mais comum de revisão foi o não funcionamento da válvula e isto é explicado pelo fato de que grande número de casos apresentava uma taxa de proteínas no líquido cefalorraqueano acima de $500 \mathrm{mg} \%$. Lombardo e Mateos ${ }^{24}$ tentaram a derivação ventrículopleural, mas dos 8 casos em que a fizeram, 5 faleceram e a necrópsia mostrou meningite séptica em todos os casos. Almeida e col. ${ }^{1}$ executaram em 16 casos a derivação ventrículo-atrial; a hipertensão intracraniana desapareceu em 10 casos e a mortalidade ocorreu em 5 casos; dêstes últimos, 4 foram submetidos à necrópsia e em nenhum havia sinais de hipertensão intracraniana.

Dos pacientes estudados neste trabalho, 33 foram submetidos à craniectomia de fossa posterior, 17 à derivação intracraniana e 20 à derivação extracraniana. Levando-se em conta o período de 30 dias de pós-operatório, em que todos os doentes puderam ser acompanhados, obteve-se melhora da hipertensão intracraniana em apenas 18 dos casos submetidos à abordagem direta e em 9 dos casos de derivação intracraniana, enquanto que nos casos submetidos à derivação extracraniana, sòmente um não melhorou. A recuperação nestes casos foi, portanto, de 95,24\%. Três casos submetidos à craniectomia tiveram que ser reoperados por apresentarem novos surtos de hipertensão intracraniana e, um dos de derivação intracraniana, por meningite. Complicações graves ocorreram em maior número nas craniectomias (11 casos), enquanto que nas derivações intracranianas registraram-se 5 casos; nas deri- 
vações extracranianas apenas dois casos tiveram complicções graves. A maior mortalidade deu-se nos casos de derivação intracraniana $(44,44 \%)$, ao passo que nos de abordagem direta, foi de $27,27 \%$, contrastando com os casos de derivação extracraniana em que não houve mortes. Observando-se o resultado nos casos que vieram a falecer e foram submetidos à necrópsia, verifica-se que em todos êles havia um processo difuso; em 4 casos, cuja cirurgia havia sido a extirpação de cisticerco de IV ventrículo, foi encontrada aracnoidite crônica e, em três dêles havia também outros cisticercos.

Mesmo não tendo valor real, em virtude do grande número de pacientes sem seguimento, foram computados neste trabalho os dados de reoperações, complicações e mortalidade, durante todo o período evolutivo. As reoperações realizadas nos casos de abordagem direta ( 5 casos) foram devidas à hipertensão intracraniana, enquanto que nos casos de derivação extracraniana (3 casos), sòmente um foi reoperado por hipertensão intracraniana; outro apresentou meningite e o terceiro, hipotensão do líquido cefalorraqueano. $O$ único caso de derivação intracraniana reoperado, o foi por meningite. Complicações graves ocorreram em maior número nos casos de abordagem direta. A maior mortalidade registrou-se nos casos submetidos à derivação intracraniana, seguida dos de abordagem direta; o menor índice foi encontrado nos de derivação extracraniana.

Considerando-se os dados de evolução e de necrópsia, conclui-se que, a neurocisticercose é um processo generalizando e que, o melhor método de tratamento cirúrgico são as derivações extracranianas.

\section{RES U M O}

A cisticercose, um dos mais sérios problemas parasitológicos do sistema nervoso, apresenta, quando localizada na fossa posterior, um quadro clínico dramático, no qual predomina a hipertensão intracraniana. Foram estudados neste trabalho, 70 pacientes com cisticercose de fossa craniana posterior, atendidos no Serviço de Neurocirurgia do Hospital das Clínicas da Faculdade de Medicina da Universidade de São Paulo de 1945 a 1968.

Considerando-se a grande diversidade existente em tôrno das técnicas de tratamento cirúrgico, foi objetivo dêste trabalho o estudo dos resultados obtidos nestes pacientes, nos quais várias técnicas foram empregadas. As cirurgias paliativas que derivam o trânsito do líquido cefalorraqueano para regiões extracranianas, quando comparadas aos outros tipos de cirurgias utilizados, foram as que proporcionaram maior índice de recuperação, exigiram menos reoperações, além de terem sido acompanhadas de menor número de complicações, bem como de menor mortalidade pós-operatória. Por outro lado, a neurocisticercose geralmente é um processo difuso, encontrando-se parasitas em várias regiões do encéfalo e/ou aracnoidite, conforme comprovou-se, também, entre os casos ora reunidos e que vieram a falecer. Baseando-se nestes fatos, não se justificam as derivações intracranianas e, a não ser eventualmente, a abordagem direta do parasita. Os casos estudados permitem cons- 
tatar, portanto, que as derivações extracranianas, por sua simplicidade e eficácia, apresentam-se, atualmente, como a terapêutica cirúrgica mais propriada à cisticercose de fossa craniana posterior.

\section{S U M M A R Y}

Surgical treatment of cysticercosis in posterior cranial fossa

Cysticercosis is one of the most severe parasitic diseases of the nervous system. When located in the posterior fossa, it presents a dramatic picture of intracranial hypertension. Seventy patients of cysticercosis in posterior cranial fossa have been studied, all of them attended at the Neurosurgery Service of the University of São Paulo Medical School, from 1945 to 1968.

Owing to the great differences in surgical procedures, it has been the objective of this work to study the results according to the technique employed. The palliative surgeries that deviate the flow of the cerebrospinal fluid to extracranial regions were the kind of surgery that gave a ketter rate of recovery, demanded less re-operations and caused not only a less amount of trouble, but also of pos-operative deaths. Neurocysticercosis is usually a spreading process, parasites being found in several regions of the brain. So, intracranial shunts cannot be justified, unless, sometimes, for the direct removal of the parasite. The cases studied allow us to state that extracranial shunts, by their simplicity and effectivenss are at present the most convenient surgical procedure in the treatment of cysticercosis of the posterior cranial fossa.

\section{$R E F E R E N C I A S$}

1. AlmeidA, G. M.; PEREIRA, W. C. \& FACURE, N. O. - Ventriculo-auriculostomia nos bloqueios ao trânsito do liquido cefallorraqueano na cisticercose encefálica. Arq. Neuro-Psiquiat. (São Paulo) 24:163, 1966.

2. AlMEIDA, G. M.; PEREIRA, 'W. C. \& FACURE, N. O. - Complicações infecciosas da ventrículo-auriculostomia. Arq. Neuro-Psiquiat. (São Paulo) 24:169, 1966.

3. ARANA-INIGUEZ, R. \& ASENJO, A. - Ventriculographic diagnosis of cysticercosis of the posterior fossa. J. Neurosurg. 2:181, 1945.

4. ARANA-IÑIgUEZ, R.; RAMOS, N. F.; FOLlE, L. E. \& GURRI, J. - Cisticercosis del 4.0 ventriculo. An. Inst. Neurol. (Montevideo) 10:95, 1953-54.

5. ARRIAGADA, C.; OJEDA, H. \& CORNEJO, J. - Clínica de la neurocisticercosis. Manifestaciones neurológicas de la cisticercosis cerebral. Neurocirurgia $19: 248,1961$.

6. ARRIAGAdA, C.; POBleTE, R.; VAlladares, H. \& HUDSON, H. - Cisticercosis ventricular: estudio clínico y evaluación del tratamiento quirúrgico en 28 casos de cisticercosis del cuarto ventriculo. Neurocirugía 19:205, 1961.

7. ARSENI, C. \& SAMITCA, D. C. - Cysticercosis of the brain. British med. J. $2: 494,1957$.

8. ASENJO, A. - Setenta y dos casos de cisticercosis en el Instituto de Neurocirugia. Rev. Neuropsiquiat. (Lima) 13:348, 1950.

9. ASENJO, A. \& ROCCA, E. D. - Compromiso de los pares craneanos en la cisticercosis cerebral. Rev. Med. (Santiago) 54:605, 1946. 
10. ASSIS, J. L. \& TENUTO, R. A. - Cisticerco racemoso intraventricular. Extirpação cirúrgica. Arq. Neuro-Fsiquiat. (São Paulo) 6:247, 1948.

11. CANELAS, H. M. - Neurocisticercose: incidência, diagnóstico e formas clinicas. Arq. Neuro-Psiquiat. (Sāo Paulo) 20:1, 1962.

12. CANELAS, H. M. - Cisticercose do sistema nervoso central. Rev. Med. (São Paulo) 47:75, 1963.

13. CANELAS, H. M.; CRUZ, O. R. \& TENUTO, R. A. - Neurocisticercose: formas clinicas pouco frequientes. Formas do ângulo pontocerebelar. Arq. Neuro-Psiquiat. (São Paulo) 20:101, 1962.

14. CARDENAS, J. C. - Cysticercosis of the nervous system: pathologic and radiologic findings. J. Neurosurg. 19:635, 1962.

15. DORFSMAN, J. - The radiologic aspects of cerebral cysticercosis. Acta radiol. $1: 836,1963$.

16. FORJAZ, S. V. \& MARTINEZ, M. - Formas obstrutivas da neurocisticercose ventricular. Arq. Neuro-Psiquiat. (São Paulo) 19:16, 1961.

17. FORJAZ, S. V.; MARTELLI, N \& LATUF, N. - Hypothalamic ventriculostomy with catheter. J. Neurosurg. 29:655, 1968.

18. FORREST, D. M. \& COOPER, D. G. W. - Complications of ventriculo-atrial shunts. A review of 455 cases. J. Neurosurg. 29:506, 1968.

19. GONI, P. B. - Cysticercosis of nervous system: clinical findings and treatment. J. Neurosurg. 19:641, 1962.

20. HAINING, R. B. \& HAINING, R. G. - Cysticercosis cerebri. J. Am. med. Ass. $172: 2 C 36,1960$.

21. IIZUKA, H. - Observaciones clínicas sobre la neurocisticercosis. Tese. Paz Montalvo, Madrid, 1961.

22. ISAMAT DE LA RIVA, F. - Cisticercosis cerebral. Vergara, Barcelona, 1957.

23. LAFON, R.; GROS, C.; LABAUGE, R.; VLAHOVITCH, B. \& RIBSTEIN, M. A propos de trois cas de cysticercose du névraxe. Rev. neurol. (Paris) 96:9, 1957.

24. LOMBARDO, L. \& MATEOS, J. H. - Cerebral cysticercosis in Mexico. Neurology (Minneapolis) 11:824, 1961.

25. LONGO, P. W.; ZUKERMAN, E.; MOREIRA, M. H. F. R.; LIMA, J. G. C.; PUPO, P. P.; LONGO, R. H.; JORDY, C. \& ZORLINI, G. - Aspectos electrencefalográficos da cisticercose encefálica. Arq. Neuro-Psiquiat. (São Paulo) 17:357, 1959.

26. MAC-CLURE, J. S. R. \& PARENTINI, L. G. - Las alteraciones cocleo-vestibulares en la cisticercosis cerebral. Neurocirugia 19:271, 1961.

27. OBRADOR, S. - Procesos expansivos de la fosa posterior. In S. Obrador \& J. S. Ibañez - Tumores Intracraniales. Paz Montalvo, Madrid, 1955, pp. 353.

28. PROCTOR, E. M.; POWELL, S. J. \& ELSDON-DEW, R. - The serological diagnosis of cysticercosis. Ann. trop. Med. Parasit. 60:146, 1966.

29. PUPO, P. P. - Cysticercosis of the nervous system. Clinical manifestations. Rev. Neurcpsiquiat. 27:70, 1964.

30. PUPO, P. P.; CARDOSO, W.; REIS, J. B. \& SILVA, C. P. — Sôbre a cisticercose encefálica: estudo clínico, anátomo-patológico, radiológico e do líquido cefalorraqueano. Arch. Serv. Assist. Psicopatas São Paulo 10:3, 1945-46.

31. RICHLAND, J. K. - Parasitic cyst of the temporal lobe with associated auditory hallucinations. Bull. Los Angeles neurol. Soc, 19:114, 1954.

32. SIMMS, N. M.; MAXWELL, R. E.; CHRISTENSON, P. C. \& FRENCH, L. A. - Internal hydrocephalus seccndary to cysticercosis cerebri: treatment with a ventriculoatrial shunt. J. Neurosurg. 30:305, 1969.

33. SPINA-FRANCA, A. - Eletroforese das proteinas do liquido cefalorraqueano na cisticercose do sistema nervoso central. Arq. Neuro-Psiquiat. (São Paulo) 18:301, 1960.

34. SPINA-FRANÇA, A. - Aspectos biológicos da neurocisticercose: alterações do liquido cefalorraqueano. Arq. Neuro-Psiquiat. (São Paulo) 20:17, 1962.

35. SPINA-FRANCA, A. - Cisticercose do sistema nervoso central. In H. M. Canelas - Manual de Clínica Neurológica. Sarvier, São Paulo, 1967, pp. 236. 
36. STEPIEN, L. - Cerebral cysticercosis in Poland. Clinical symptoms and operative results in 132 cases. J. Neurosurg. 19:505, 1962.

37. TOLOSA, E. - Expérience neuro-chirurgicale sur les hydrocéphalles par cysticercose. Considérations sur 10 cas. Rev. Neurol. (Paris) 82:441,1950.

38. TOLOSA, E. - Cysticercose cérébrale: aspects cliniques et possibilités thérapeutiques. Rev. Neurol. (Paris) 90:187, 1954.

39. VAllaDARES, H.; CONTRERAS, M. \& DONOSO, M. - Cisticercosis cerebral. Criterio quirúrgico. Neurocirugia 8:61, 1951.

40. VAlLADARES, H. \& POBLETE, R. - Tratamiento quirúrgico de la cisticercosis cerebral. Neurocirugía 19:286, 1961.

41. VARIETA, O. J.; OBERHAUSER, A. E. \& WEINSTEIN, C. W. - Contribuición al estudio bioquímico de la neurocisticercosis. Neurocirugia 19:280, 1961.

42. ZACLIS, J. - Contribuição radiológica para o diagnóstico da cisticercose. Rev. paul. Med. 43:165, 1953.

Rua Pernambuco 648 - Londrina, $P R-$ Brasil. 